\title{
Construction practice of student evaluation system based on JFinal + webix integrated framework and Baidu Al platform
}

\author{
Zhan Gao ${ }^{1, *}$, Zhihai Suo ${ }^{1}$, Jun $\mathrm{Liu}^{1}$, Mo Xu${ }^{1}$, Dandan Hong ${ }^{1}$, Hua Wen ${ }^{1}$, and Xiangting $\mathrm{Ji}^{2}$ \\ ${ }^{1}$ Xi' an Jiao Tong university, Networdk information center, 29 Xianning West Road, China \\ ${ }^{2}$ Baidu Inc., Baidu Campus, No. 10 Shangdi 10th Street, Haidian Distrlct, Beijing 100085, P.R.China
}

\begin{abstract}
Students' evaluation of teaching is a key link to realize teaching quality monitoring and promote teachers' teaching level. Based on the practice of student evaluation in our university, this paper constructs a multi-level student evaluation system, and develops an online student evaluation system by using JFinal+webix integration framework. The new Internet plus evaluation model is established to improve the efficiency of student evaluation and the enthusiasm of students to evaluate teaching. Meanwhile, based on the analysis of students' comments on teaching by Baidu AI platform, It provides data support for the improvement of learning level and the optimization of teaching evaluation.
\end{abstract}

\section{Introduction}

In the process of the popularization of higher education, the most prominent problem is how to ensure the quality of teaching. Teaching quality is an important lifeline related to the survival and development of colleges and universities 1. As the basic form of higher education activities, classroom teaching is in the leading position under the current teaching mode. Therefore, it is very important to establish an efficient, standardized and effective classroom teaching quality evaluation for the monitoring of teaching quality in Colleges and universities. Among all the teaching quality evaluation activities, the most vivid and effective is "student evaluation of teaching" 2 .

The original student evaluation of teaching work mostly adopts paper filling questionnaires, which is time-consuming and laborious and easy to make mistakes. Data can not be effectively accumulated. With the development of Web technology and the construction of intelligent campus in universities, colleges and universities have gradually abandoned traditional student evaluation methods, and adopted the Internet plus evaluation method to build a new student evaluation mode. This mode can be completed efficiently. At the same time, because of the convenience of online teaching evaluation and greatly improved the enthusiasm of students in teaching evaluation, the data of students' evaluation of teaching can also be accumulated, saved and used.

* Corresponding author: zgao@mail.xjtu.edu.cn 
Xi'an Jiaotong University has a history of more than 120 years and excellent undergraduate teaching tradition. It always adheres to the core value of cultivating morality and talents, and adheres to the central task and main responsibility of talent cultivation. The school attaches great importance to the improvement of education and teaching quality, and has carried out a large number of empirical research and pilot application work in the aspects of innovative teaching mode and method, teaching quality system guarantee reform, etc. in order to actively respond to the school's innovative measures in student evaluation of teaching, it is urgent to establish an efficient and convenient online student evaluation system to realize multi-level learning At the same time, based on Baidu AI platform, emotional analysis, processing and classification of students' teaching evaluation opinions are carried out to form teachers' evaluation summary, which provides important data support for the improvement of school teaching quality.

\section{Overall construction requirements of student evaluation system}

Through the establishment of information collection, evaluation and feedback mechanism, data services are provided to management departments, teachers and students, and a realtime and dynamic student evaluation and management system is established with accurate quantification, normal continuity, comprehensive coverage and real-time dynamic.

The main functions of the platform are as follows:

1. Students: use PC / mobile phone client to realize online teaching evaluation function, which can view the evaluation results in real time, and ensure the objectivity and realtime of teaching evaluation data.

2. Teachers: teachers can check the teaching evaluation results and opinions at any time, realize self-examination, self-evaluation and self correction, and adjust teaching methods and methods in time.

3. Leaders and management departments of the school and College: through data statistics and chart analysis, provide the overall situation of students' evaluation of teaching for the leaders and management departments of the school and college, provide data support for the decision-making of schools, colleges and management departments, and improve the scientific level of educational decision-making. At the same time, we use Baidu AI platform to help teaching diagnosis, and locate the classroom by analyzing students' teaching evaluation opinions There are some problems in teaching, so as to promote teachers to attach importance to teaching and support the teaching quality assurance of schools and the analysis of teachers' learning situation.

\section{Design and implementation of the system}

The design and implementation of the system starts from the content and process of teaching evaluation, constructs a multi-dimensional student evaluation system under the background of smart campus, combines the data of graduate school, academic affairs office, personnel department and other business departments, constructs online student evaluation system, realizes in-depth analysis and mining of student evaluation data, timely finds, tracks, feeds back and gradually solves classroom teaching Learn related questions.

\subsection{System architecture design}

This system is based on JFinal + webix integrated framework. JFinal framework is 
developed rapidly, with less code, powerful function, lightweight and easy to expand. The front-end uses advanced webix framework, which is a cross browser, cross platform JavaScript framework, built-in rich components, supports HTML5 and CSS3, has good compatibility with various browsers and improves user experience. Through this lightweight integration framework, we can realize the transmission, storage, processing and control of the information flow of students' evaluation of teaching, and realize the convenient, fast, efficient and accurate automatic management.

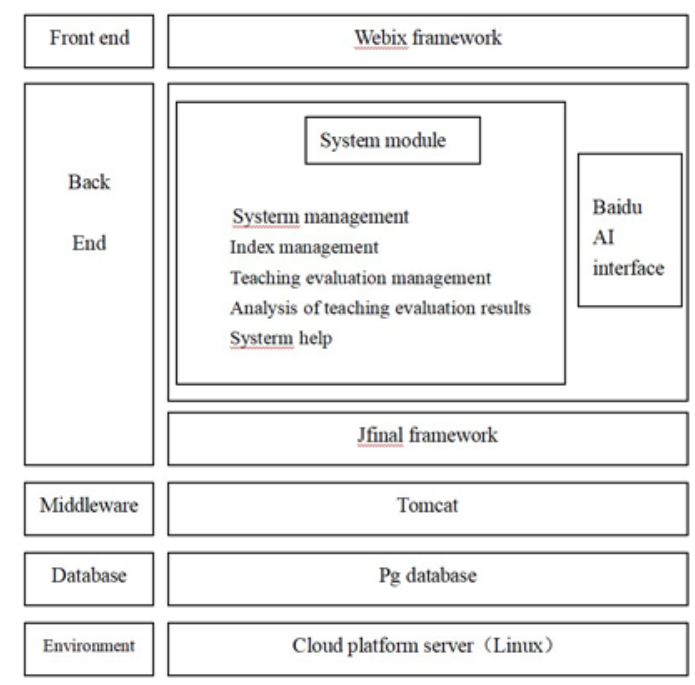

Fig. 1. System architecture.

\subsection{Function design of the system}

The system mainly includes five modules: index management, student evaluation, result analysis, system management and system help.

1) Index management: the index system of student evaluation of teaching is divided into three levels: School index, college index and teacher index. The graduate school is responsible for maintaining the school indicators. Both the college index and the teacher index are open indicators, and each can set a question.

2) Teaching evaluation management: during the opening period of teaching evaluation, after students $\log$ in through unified identity authentication, online scoring and teaching evaluation are carried out for each class according to the index system. The system automatically calculates the score according to the weight, and it is available for students to view and modify.

3) Analysis of teaching evaluation results: each semester, the teaching evaluation results are analyzed and displayed from different dimensions of students, courses, teachers, etc., and the students' teaching evaluation opinions are intelligently analyzed by PaddlePaddle framework to form a summary of teaching evaluation opinions at different levels of teachers, colleges and schools. Teachers,colleges and administrators can view the teaching evaluation analysis results according to their authority.

4) System management: realize the management of users, roles, permissions, resources, logs, organizational structure and other important parameters. It is mainly open for administrators to configure and manage the system flexibly.

5) System help: provide relevant system help documents for users of various roles. 


\subsection{System security design}

Based on the protection of students' privacy in students' evaluation of teaching, the overall security protection of the system mainly includes the following four aspects:

1) Identity authentication: Based on the school cross system single sign on function, users only go through one login and identity authentication. When other systems log in later, they will automatically identify the user's identity information and login information. MD5 encryption technology is used to encrypt the user's login password to effectively avoid hacker attacks.

2) Role permission: according to the control of security rules or relevant policies, different users have different access rights. Users can access the relevant resources only belonging to their own authorization, such as the function menu of application system, buttons of each interface, columns of data display (such as menu or page resources).

3) MAC address access control: for computers accessing the core functions of the system, MAC address binding authentication is strictly implemented. Only authorized computers can log in to the system and manage the core functions.

4) Page security: the access of each page is strictly checked. When the address is directly entered, the system will automatically detect the user login, role and permission information to ensure the legitimacy of page access. The security of the input data is checked to avoid the occurrence of SQL injection.

\subsection{Core code implementation}

\subsubsection{JFinal core configuration}

This system adopts JFinal technology, which uses a global filter to filter requests and is responsible for initialization of all JFinal contents. The filter code added to the web.xml,the coed is as follows:

$<$ filter $>$

$<$ filter-name $>$ jfinal $</$ filter-name $>$

$<$ filter-class $>$ com.jfinal.core.JFinalFilter $</$ filter-class $>$

$<$ init-param $>$

$<$ param-name $>$ configClass $<$ /param-name $>$

$<$ param-value $>$ com.gste.scoreConfig $<$ /param-value $>$

$</$ init-param $>$

$</$ filter $>$

$<$ filter-mapping $>$

$<$ filter-name $>$ jfinal $</$ filter-name $>$

$<$ url-pattern $>/ *<$ url-pattern $>$

$</$ filter-mapping $>$

Based on the consideration of data security and complexity, the database adopts PostgreSQL database. The key information of database link is encrypted in the database link configuration. Even if you get the configuration file, you can't directly obtain the user name, password and other key information.

JFinal directly maps each field in the data table to the entity through its model file. It can be transferred in the form of entity data and can be called in the page form. Next, taking the evaluation result information table as an example, we first need to establish an entity class xspj. It has many convenient methods to operate the database by inheriting the model, which greatly reduces the amount of code. 


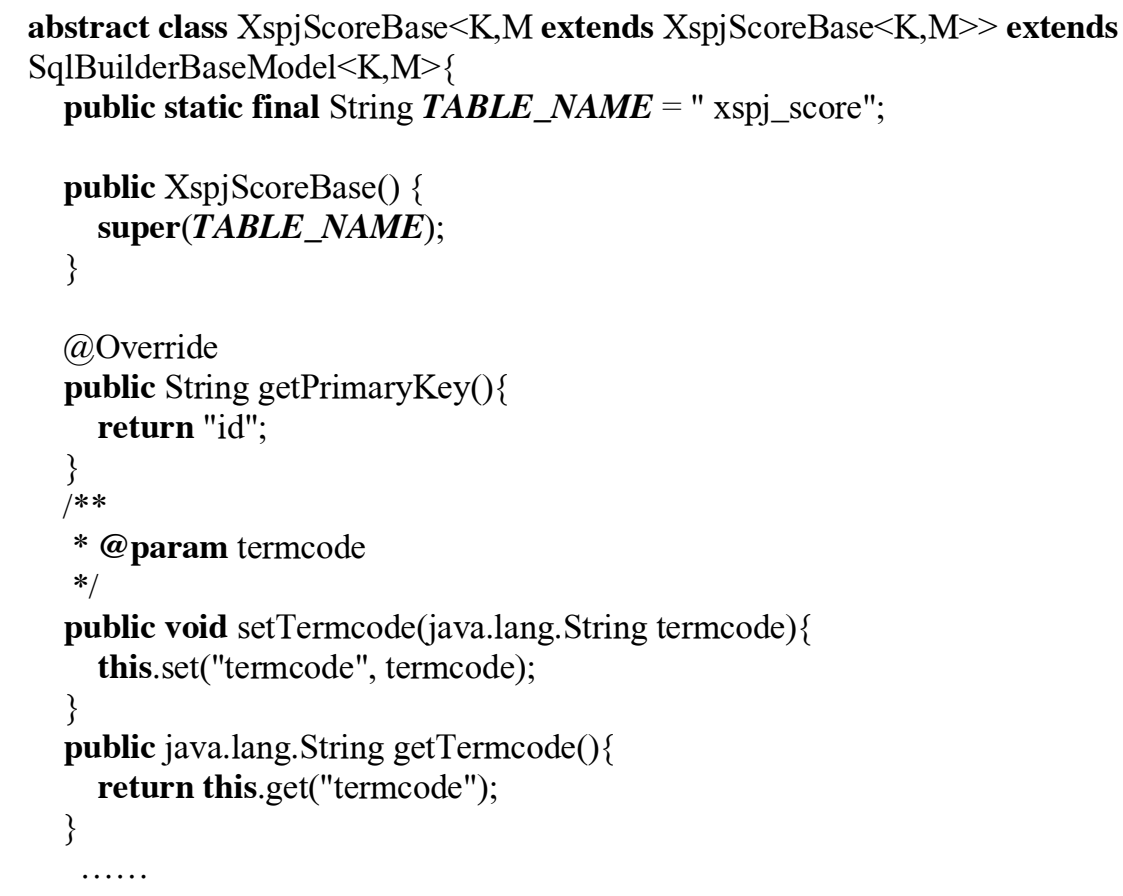

\subsubsection{Analysis of students' comments on Teaching}

The interface of PaddlePaddle framework is used for the analysis of students' teaching evaluation opinions. It can realize the word segmentation, part of speech tagging and emotional analysis of teaching evaluation opinions, eliminate ambiguity and support the accurate understanding of natural language. The system has built-in call interface, which needs to input app_ ID, API_Key and secret_Key, easy to use at any time.

public class TextAPI \{

public static final String APP_ID = "**";

public static final String API_KEY = "**";

public static final String SECRET_KEY = "**";

public static void main(String[] args) \{

AipNlp client $=$ new AipNlp(APP_ID, API_KEY, SECRET_KEY);

client.setConnectionTimeoutInMillis(2500);

client.setSocketTimeoutInMillis(70000);

client.setHttpProxy("proxy_host", proxy_port);

client.setSocketProxy("proxy_host", proxy_port);

... $\}$ \}

JSONObject res = client.lexer(args, null);

At the same time, we use the word cloud in echarts to show the analysis results, which is convenient for users to understand the opinion analysis results more intuitively.

\section{Application effect}

The student evaluation system of Xi'an Jiaotong University synchronizes and integrates the data of students, teachers and courses in real time, which provides important data support for the work of teacher performance evaluation, professional title evaluation, annual 
assessment and excellent teacher selection. For teachers, they can intuitively understand students' evaluation of teaching content, teaching attitude, teaching methods and teaching effect, as well as timely understand students' suggestions and opinions on the course; for students, they can quickly submit and feedback the classroom learning situation, classroom teaching environment and classroom teaching opinions; for the management department (college, teaching In terms of the management department, it can timely understand the overall classroom teaching evaluation, the teaching effect of the course, the opinions and suggestions of the teaching environment, and the feedback of the course opening. Finally, the construction of quantitative accurate, normal continuous, comprehensive coverage, realtime dynamic benign student evaluation and feedback mechanism. After the system was officially launched in June 2018, it supports more than 20000 students to evaluate teaching each semester, and collects more than 10000 effective evaluation opinions.

\section{Conclusion}

This paper introduces the overall construction requirements of graduate teaching evaluation system in detail, analyzes and designs the system architecture, function and security in detail, and finally describes the system implementation details. The construction of the system solves the problems of low efficiency and small coverage of offline teaching evaluation, improves the overall work efficiency, and further helps teachers improve the teaching effect.

\section{References}

1. Xuan xing rong,Chen hui.Heilongjiang Education (higher education research and evaluation).11(2012).

2. Li Min,Mao Xiaoyan.Deepening the reform of management mode and establishing teaching quality monitoring system. China higher education. z2(2013) 